\title{
About de Smit's question on flatness
}

\author{
Sylvain Brochard • Ariane Mézard
}

Received: 14 June 2008 / Accepted: 16 June 2009 / Published online: 24 October 2009

(C) The Author(s) 2009. This article is published with open access at Springerlink.com

\begin{abstract}
Let $\varphi: A \rightarrow B$ be a flat morphism of Artin local rings with the same embedding dimension. Denote by $\mathfrak{m}_{A}$ the maximal ideal of $A$. Bart de Smit asked whether any finite $B$-module that is $A$-flat is $B$-flat. We prove the conjecture in embedding dimension one or two. In embedding dimension $n$, we prove the conjecture under an additional assumption on $B / \mathfrak{m}_{A} B$.
\end{abstract}

Keywords Flatness $\cdot$ Artin rings $\cdot$ Complete intersections $\cdot$ Gorenstein rings

\section{Introduction}

Notations and conventions All rings are commutative rings with unit. If $A$ is a local ring, its maximal ideal and residue field are always denoted by $\mathfrak{m}_{A}$ and $\kappa(A)$. If $M$ is an $A$-module, the minimal number of generators of $M$ is denoted by $\mu_{A}(M)$. We will say that an element of $M$ is a minimal generator of $M$ if it is part of a minimal system of generators for $M$. The embedding dimension of $A$ is the dimension of the $\kappa(A)$-vector space $\mathfrak{m}_{A} / \mathfrak{m}_{A}^{2}$. It is denoted by edim $(A)$. Note that edim $(A)$ is equal to $\mu_{A}\left(\mathfrak{m}_{A}\right)$. A morphism $\varphi: A \rightarrow B$ of local rings is a complete intersection morphism (resp. a Gorenstein morphism) if $B / \mathfrak{m}_{A} B$ is a complete intersection ring (resp. a Gorenstein ring). (Unlike [4, IV 19.3.6], a complete intersection morphism is not necessarily flat. Note also that a Gorenstein or complete intersection morphism between local rings is local by definition, since Gorenstein or complete intersection local rings are not null.)

S. Brochard (西)

Mathematisch Instituut, Universiteit Leiden, Postbus 9512, 2300 RA Leiden, The Netherlands e-mail: brochard@math.leidenuniv.nl

\section{A. Mézard}

Département de mathématiques, Université de Versailles Saint-Quentin, 45, avenue des États-Unis, 78035 Versailles Cedex, France e-mail: mezard@math.uvsq.fr 
Statement of the conjecture and results Let us first consider a very easy example. Take $A=k[x] /\left(x^{2}\right)$ and $B=A[u] /\left(u^{2}-x\right) \simeq k[u] /\left(u^{4}\right)$ (where $k$ is a field). Let $M$ be a finite $B$-module, that is free over $A$. Let $\left(e_{1}, \ldots, e_{n}\right)$ be an $A$-basis of $M$ and let $U$ be the matrix of multiplication by $u$. We can write $U=U_{0}+U_{1} x$ where $U_{0}$ and $U_{1}$ have coefficients in $k$. Then the relation $U^{2}=x$.Id yields

$$
\left\{\begin{array}{l}
U_{0}^{2}=0 \\
U_{0} U_{1}+U_{1} U_{0}=\mathrm{Id} .
\end{array}\right.
$$

Using these relations, it is an easy exercise in linear algebra over a field to verify that $n$ is necessarily even, and that $M$ is actually free over $B$.

In the 1990s, Bart de Smit raised a question: could the following generalization be true?

Conjecture 1.1 (de Smit) Let $\varphi: A \rightarrow B$ be a flat morphism of Artin local rings with the same embedding dimension. Then every $B$-module $M$ that is flat and of finite type over $A$, is flat over $B$.

Remark 1.2 Note that, since the maximal ideal of an Artin local ring is nilpotent, a morphism between to such rings is necessarily local. Recall also the following from [2, chap. II, Sect. 3, $\mathrm{n}^{\mathrm{0}} 2$, corollary 2 of proposition 5]. Let $M$ be a module over a Noetherian local ring $A$, and assume that $M$ is of finite type or that $A$ is Artin. Then it is equivalent to say that $M$ is flat, projective, or free. The case where $M$ is of finite type is well-known, the case where $A$ is Artin follows from 2.1 (iv).

To explain the results contained in our paper, we propose the following definition.

Definition 1.3 Let $\varphi: A \rightarrow B$ be a local morphism of Noetherian local rings. We say that $\varphi$ is a de Smit morphism if every $B$-module $M$ that is flat and of finite type over $A$ is flat over $B$.

Our first observation is that a morphism satisfying the hypothesis of de Smit's conjecture is necessarily a complete intersection morphism (see 2.5), hence a Gorenstein morphism. We introduce below another class of morphisms, which we call nice morphisms. The precise definition is given in 4.5 . We then prove the following results:

Theorem 1.4 (see 4.7) A nice and Gorenstein morphism of Artin local rings is de Smit.

Theorem 1.5 (see 4.10) Let $\varphi: A \rightarrow B$ be a complete intersection morphism of Noetherian local rings. Assume that $\operatorname{edim}(B)<\operatorname{edim}(A)$. Then the only $B$-module (of finite type over $A$ if $A$ is not Artin) that is flat over $A$ is zero. In particular $\varphi$ is nice and de Smit.

Theorem 1.6 (see 5.1, 5.11 for (i), see 5.12 for (ii) and 5.13 for (iii)) Let $\varphi: A \rightarrow B$ be a complete intersection morphism of Artin local rings. Assume that $A$ and $B$ have same embedding dimension $n$ and that one of the following holds.

(i) The embedding dimension $n$ is 0,1 or 2 .

(ii) $n=3$ and there is a minimal generator $u$ of $\mathfrak{m}_{B}$ that divides (in $B$ ) an element of $\mathfrak{m}_{A} B \backslash \mathfrak{m}_{B} \mathfrak{m}_{A} B$ (i.e. a minimal generator of $\mathfrak{m}_{A} B$, for instance the image of a minimal generator of $\mathfrak{m}_{A}$ if $\varphi$ is flat, see 2.5).

(iii) Let $B_{0}=B / \mathfrak{m}_{A} B$. For some minimal system of generators $u=\left(u_{1}, \ldots, u_{l}\right)$ of $\mathfrak{m}_{B_{0}}$, there is an upper triangular $u$-Wiebe matrix for $B_{0}$ (the definition of Wiebe matrices is recalled in 5.2).

Then $\varphi$ is nice (hence de Smit). 
Remark 1.7 The additional assumption given in embedding dimension 3 (in (ii)) can be replaced by any of the equivalent conditions given in Lemma 5.6. The authors initially hoped that this condition would always hold. This is unfortunately not the case as we can see in Example 6.1.3. In practice, this condition is used to reduce the statement to embedding dimension $n-1$ (see the Lemma 5.7). Actually, the condition given in (iii) more or less means that the condition discussed above "recursively holds". There is a Maple file available on the web page of the first author for testing whether this condition does, or does not, hold (in the case $n=3, \varphi$ being given with explicit equations, and with the assumption that $A$ and $B$ have residue field equal to $\mathbb{C}$ ).

Example 1.8 If in de Smit's Conjecture 1.1 we drop the condition edim $(A)=\operatorname{edim}(B)$, then there are trivial counter-examples. For instance let $A$ be a field and let $B=A[\varepsilon] /\left(\varepsilon^{2}\right)$. Then the ideal $(\varepsilon)$ of $B$ is obviously flat over $A$, and not flat over $B$.

The text is organised as follows: the first paragraph is devoted to basic consequences of Nakayama's lemma and to basic facts on embedding dimension and complete intersection rings of dimension zero (Sect. 2). There is nothing original in this section, but we remark that the hypotheses of de Smit's conjecture imply that $B$ is a relative complete intersection of dimension zero over $A$. The discussion of de Smit's conjecture is carried out in the following. First (Sect. 3) we introduce the notion of weakly torsion-free modules and give an equivalent condition for flatness over $B$ in these terms (see 3.7 and 3.9). Then (Sect.4) we define and study the flatness in first order over a Noetherian local ring $A$. It is weaker than flatness, and equivalent to flatness if $\mathfrak{m}_{A}^{2}=0$ (see 4.3). We prove the conjecture for embedding dimension less than two and the other results of 1.6 in Sect. 5. At last, we discuss some examples, some generalizations of de Smit's conjecture and possible applications (Sect.6).

\section{Flatness and complete intersections}

We prove in this section that a morphism that satisfies the hypothesis of the Conjecture 1.1 is always a complete intersection morphism. Let us first recall briefly the following useful lemma and give some consequences (note that $M$ is not necessarily of finite type).

Lemma 2.1 [2, chap. II, Sect. 3, $\mathrm{n}^{\circ}$ 2, prop. 4 and 5] Let $A$ be a ring, a an ideal that is contained in the radical of $A$, and $M$ an A-module. Assume that $\mathfrak{a}$ is nilpotent or that $M$ is of finite type.

(i) If $M=\mathfrak{a} M$ then $M=0$.

(ii) Let $N$ be a submodule of $M$. Then $M=N+\mathfrak{a} M$ implies $M=N$.

(iii) Let $\left(x_{i}\right)_{i \in I}$ be a family of elements of $M$, the images of which generate the $A / \mathfrak{a}$-module $M / \mathfrak{a} M$. Then the $x_{i}$ 's generate $M$.

(iv) Let $\left(x_{i}\right)_{i \in I}$ be a family of elements of $M$, the images of which form a basis of the $A / \mathfrak{a}$-module $M / \mathfrak{a} M$. Assume that $M$ is flat over $A$. Then the $x_{i}$ 's form a basis of $M$.

Remark 2.2 In the whole paper, the finiteness assumption on the module $M$ is only used in Nakayama's lemma. Thus it is useless if $A$ is Artin, and in this case we can remove the sentence "of finite type over $A$ " in the Definition 1.3 of de Smit morphisms.

Corollary 2.3 Let $\varphi: A \rightarrow B$ be a local morphism of Noetherian local rings. Let $M$ be a $B$-module, flat over A. Assume that $M$ is of finite type over $B$ or that $A$ is Artin. Then the following conditions are equivalent. 
(i) The module $M$ is flat over $B$.

(ii) The module $M / \mathfrak{m}_{A} M$ is flat over $B / \mathfrak{m}_{A} B$.

Proof (i) $\Rightarrow$ (ii) is obvious, since $M / \mathfrak{m}_{A} M$ is isomorphic to $M \otimes_{B}\left(B / \mathfrak{m}_{A} B\right)$. Conversely, let $\left(f_{i}\right)_{i \in I}$ be a family of elements of $M$, the images of which in $M / \mathfrak{m}_{A} M$ form a basis of $M / \mathfrak{m}_{A} M$ over $B / \mathfrak{m}_{A} B$. We will prove that the $f_{i}$ 's form a basis of $M$ over $B$. We already know that they generate $M$ (use 2.1 with the ideal $\mathfrak{a}=\mathfrak{m}_{A} B$ ). Let us consider the short exact sequence

$$
0 \longrightarrow K \longrightarrow B^{(I)} \stackrel{\psi}{\longrightarrow} M \longrightarrow 0
$$

where $\psi$ is given by $\left(\lambda_{i}\right) \mapsto \sum \lambda_{i} f_{i}$ and $K$ is its kernel. Since $M$ is flat over $A$, this sequence remains exact after applying the functor.$\otimes_{A}\left(A / \mathfrak{m}_{A}\right)$. Thus $K \otimes_{A}\left(A / \mathfrak{m}_{A}\right)=0$. Applying 2.1 to the $B$-module $K$ and the ideal $\mathfrak{m}_{A} B$ (note that if $M$ is of finite type over $B$, then $I$ is finite and $K$ is of finite type over $B$ as well) we deduce that $K=0$, proving that $\psi$ is an isomorphism.

Corollary 2.4 To prove the Conjecture 1.1, we can assume that the maximal ideal $\mathfrak{m}_{A}$ of $A$ is a square-zero ideal.

Proof Assume that the conjecture is true with the additional hypothesis $\mathfrak{m}_{A}^{2}=0$. Let $M$ be an $A$-flat $B$-module. Using our assumption and tensoring by $A / \mathfrak{m}_{A}^{2}$, we see that $M / \mathfrak{m}_{A}^{2} M$ is a flat $\left(B / \mathfrak{m}_{A}^{2} B\right)$-module. Now tensoring by $A / \mathfrak{m}_{A}$, we deduce that $M / \mathfrak{m}_{A} M$ is flat over $B / \mathfrak{m}_{A} B$. Hence $M$ is flat over $B$ owing to 2.3 .

Proposition 2.5 Let $\varphi: A \rightarrow B$ be a flat local morphism of Noetherian local rings.

(i) The $B / \mathfrak{m}_{A} B$-module $\mathfrak{m}_{A} B / \mathfrak{m}_{A}^{2} B$ is free, and

$$
\operatorname{rk}_{B / \mathfrak{m}_{A} B} \frac{\mathfrak{m}_{A} B}{\mathfrak{m}_{A}^{2} B}=\operatorname{dim}_{\kappa(B)} \frac{\mathfrak{m}_{A} B}{\mathfrak{m}_{B} \mathfrak{m}_{A} B}=\operatorname{edim}(A) .
$$

(ii) We have edim $(A) \leq \operatorname{edim}(B)$.

(iii) Assume moreover that $\operatorname{edim}(B) \leq \operatorname{edim}(A)+1$. Then $\varphi$ is a complete intersection morphism.

Proof (i) First we note that, as $B$ is flat over $A$, we have canonical isomorphisms:

$$
\begin{aligned}
\mathfrak{m}_{A} B / \mathfrak{m}_{A}^{2} B & \simeq\left(\mathfrak{m}_{A} / \mathfrak{m}_{A}^{2}\right) \otimes_{A} B \\
& \simeq\left(\mathfrak{m}_{A} / \mathfrak{m}_{A}^{2}\right) \otimes_{\kappa(A)}\left(B / \mathfrak{m}_{A} B\right)
\end{aligned}
$$

so that $\mathfrak{m}_{A} B / \mathfrak{m}_{A}^{2} B$ is free of rank edim $(A)$ over $B / \mathfrak{m}_{A} B$. We also have

$$
\begin{aligned}
\left(\frac{\mathfrak{m}_{A} B}{\mathfrak{m}_{B} \cdot\left(\mathfrak{m}_{A} B\right)}\right) & \simeq\left(\mathfrak{m}_{A} B\right) \otimes_{B} \kappa(B) \\
& \simeq\left(\mathfrak{m}_{A} B\right) \otimes_{B}\left(B / \mathfrak{m}_{A} B\right) \otimes_{\left(B / \mathfrak{m}_{A} B\right)} \kappa(B) \\
& \simeq\left(\mathfrak{m}_{A} B / \mathfrak{m}_{A}^{2} B\right) \otimes_{\left(B / \mathfrak{m}_{A} B\right)} \kappa(B)
\end{aligned}
$$

so that the dimension of $\frac{\mathfrak{m}_{A} B}{\mathfrak{m}_{B} \cdot\left(\mathfrak{m}_{A} B\right)}$ over $\kappa(B)$ is also equal to edim $(A)$.

(ii) and (iii). If $A$ and $B$ are Artin, the ideal $\mathfrak{m}_{A} B$ is $\mathfrak{m}_{B}$-primary and we can apply $[12,2.1$ and 2.3]. In the general case, we will see that the same proofs (more or less) still work. First we replace $\varphi$ by its composition with the natural map from $B$ into its completion for its 
$\mathfrak{m}_{B}$-adic topology. This affects neither the hypothesis, nor the conclusions. We are thus reduced to the case where $B$ is a complete Noetherian local ring. With the Cohen structure theorem for complete Noetherian local rings we now have a surjective map $\pi: R \rightarrow B$ where $R$ is a regular local ring with $\operatorname{edim}(B)=\operatorname{edim}(R)$. Write $I=\pi^{-1}\left(\mathfrak{m}_{A} B\right)$ and $J=\pi^{-1}\left(\mathfrak{m}_{A}^{2} B\right)$. We have $I \supset J \supset I^{2}$ and $R / I \simeq B / \mathfrak{m}_{A} B$. Owing to (i), we also have that $I / J \simeq \mathfrak{m}_{A} B / \mathfrak{m}_{A}^{2} B$ is a free $R / I$-module of rank $r=\operatorname{edim}(A)$. Since every ideal in a regular local ring has finite projective dimension, we conclude with [12, Theorem 1.1] that $I=\left(x_{1}, \ldots, x_{r}\right)+J$, where $\left(x_{1}, \ldots, x_{r}\right)$ is a regular sequence in $R$. As obviously $r \leq \operatorname{dim}(R)=\operatorname{edim}(R)$, we obtain (ii).

With the remark in [12] before its Corollary 1 , we also know that the module $I /\left(x_{1}, \ldots, x_{r}\right)$ has finite projective dimension over $R /\left(x_{1}, \ldots, x_{r}\right)$.

In the case $\operatorname{edim}(A)=\operatorname{edim}(B)$, that is, in the case $r=\operatorname{edim}(R)$, the sequence $\left(x_{1}, \ldots, x_{r}\right)$ is a maximal regular sequence in $R$. This implies that $\operatorname{dim}\left(R /\left(x_{1}, \ldots, x_{r}\right)\right)=0$ and, as in [12], Proof of Theorem 2.3, we observe that the ideal of finite projective dimension $I /\left(x_{1}, \ldots, x_{r}\right)$ of $R /\left(x_{1}, \ldots, x_{r}\right)$ must be null (remember that a nonnull ideal of finite projective dimension always contains a regular element). Thus $I=\left(x_{1}, \ldots, x_{r}\right)$, so that $R / I \simeq$ $B / \mathfrak{m}_{A} B$ is a complete intersection of dimension zero. In the case edim $(B)=\operatorname{edim}(A)+1$, we refer to [12] and its Proof of Theorem 2.3 again to reach the conclusion.

\section{Weakly torsion-free modules}

Let $\varphi: A \rightarrow B$ be a Gorenstein morphism of Artin local rings. Let us consider a $B$-module $M$ that is flat over $A$. We will give a necessary and sufficient condition for the module $M$ to be flat over $B$ (3.9). For that purpose, we introduce the notion of weakly torsion-free modules.

Definition 3.1 Let $R$ be a local ring and $M$ be an $R$-module. We say that $M$ is weakly torsion-free if, for every $\lambda$ in $R$ and every $m$ in $M$, the relation $\lambda m=0$ implies $\lambda=0$ or $m \in \mathfrak{m}_{R} M$.

The main result of this section is the flatness criterion 3.7 for modules over a Gorenstein, Artin, local $k$-algebra. First, we need the following lemma.

Lemma 3.2 Let $k$ be a field and $R$ an Artin local $k$-algebra. Assume that $R$ is Gorenstein and that its residue field is $k$. Then there exists a basis $\left(e_{1}, \ldots, e_{n}\right)$ of $R$ as a $k$-vector space, together with elements $a_{1}, \ldots, a_{n-1}$ of $R$ such that the following holds.

1. for all $i$ we have $a_{i} e_{i}=e_{n}$

2. for all $j>i, a_{i} e_{j}=0$.

Example 3.3 For the $k$-algebra $k[u, v] /\left(u^{3}, v^{3}\right)$, the basis

$$
\left(1, u, u^{2}, v, u v, u^{2} v, v^{2}, u v^{2}, u^{2} v^{2}\right)
$$

works. We can take as $a_{i}$ 's the elements $u^{2} v^{2}, u v^{2}, v^{2}, u^{2} v, u v, v, u^{2}, u$.

Remark 3.4 Recall that, in a Gorenstein local ring $R$ of dimension 0 , there is a unique minimal ideal, called the socle of $R$. If $\mathfrak{m}_{R}$ is the maximal ideal of $R$, then the socle of $R$ is equal to the annihilator $\operatorname{Ann}\left(\mathfrak{m}_{R}\right)$ of $\mathfrak{m}_{R}$ (see for example [11] for a formal computation of this annihilator). Now, if $\left(e_{1}, \ldots, e_{n}\right)$ is as in the lemma, then $e_{n}$ is necessarily a generator of the socle of $R$. Indeed, $e_{n}$ is different from zero since it is part of a basis of $R$, and if $v$ is a nonzero element of $R$, we can write $v=\alpha_{i} e_{i}+\cdots+\alpha_{n} e_{n}$ with $\alpha_{i}$ an invertible element. 
Multiplying by $a_{i}$, we see that $e_{n}$ belongs to $(v)$, so that the principal ideal generated by $e_{n}$ is the minimal ideal of $R$.

Moreover, the element $e_{1}$ is necessarily invertible in $R$, and this is the only one. Indeed, we know that $a_{1} e_{1}$ is different from zero, so that $a_{1} \neq 0$. But we also have $a_{1} e_{j}=0$ for all $j>1$ so that the $e_{j}$ 's cannot be invertible for $j \geq 2$. It follows that $e_{1}$ is invertible, since the basis necessarily contains at least one invertible element.

Proof Let $e_{n}$ be a generator of the socle of $R$, and let us take elements $e_{1}, \ldots, e_{n-1}$ such that $\left(e_{1}, \ldots, e_{n}\right)$ is a basis of $R$. We proceed by induction. Assume that we already have constructed elements $a_{1}, \ldots, a_{i-1}$ with the properties of the lemma $(1 \leq i \leq n-1)$. As $e_{n}$ is in the socle of the ring, there exists an element $a_{i}$ such that $a_{i} e_{i}=e_{n}$. Note that $a_{i}$ is not invertible, so $a_{i} e_{n}=0$. If there is an $e_{j}, j>i$ such that $a_{i} e_{j}$ does not belong to $\left(e_{n}\right)$, then we exchange $e_{i}$ and $e_{j}$ and we replace $a_{i}$ with some multiple of $a_{i}$ in such a way that $a_{i} e_{i}$ is still equal to $e_{n}$. Now, for every $j>i, a_{i} e_{j}$ belongs to $\left(e_{n}\right)$, so we can write $a_{i} e_{j}=\lambda_{j} e_{n}$. We replace $e_{j}$ with $e_{j}-\lambda_{j} e_{i}$. This gives the result.

Remark 3.5 The Gorenstein assumption cannot be removed. Let $R$ be equal to $k[x, y] /$ $(x, y)^{2}=k[x, y] /\left(x^{2}, x y, y^{2}\right)$. Assume that there exists a basis $\left(e_{1}, e_{2}, e_{3}\right)$ and elements $a_{1}, a_{2}$ as in the lemma. We have seen in the preceding remark that $e_{2}$ is in the maximal ideal $\mathfrak{m}_{R}$ of $R$. Moreover, the relation $a_{2} e_{3}=0$ proves that $a_{2}$ is also in the maximal ideal. Then $a_{2} e_{2}=0$, which yields a contradiction.

Remark 3.6 The hypothesis on the residue field cannot be removed. For instance if $R$ is a finite (nontrivial) extension field of $k$, the lemma is obviously false for $R$.

Proposition 3.7 Let $R$ be an Artin local ring. Assume that $R$ is Gorenstein and contains a field. Then an $R$-module $M$ is flat over $R$ if and only if it is weakly torsion-free.

Proof Assume that $M$ is flat over $R$. Let $\lambda$ be a nonzero element in $R$ and $m \in M$ such that $\lambda m=0$. As $M$ is flat over $R$, we can write $m=\sum_{i} \alpha_{i} e_{i}$ where the $e_{i}$ 's belong to an $R$-basis of $M$. Thus, for every $i, \lambda \alpha_{i}=0$, so that $\alpha_{i}$ cannot be invertible and belongs to $\mathfrak{m}_{R}$.

Conversely, assume that $M$ is weakly torsion-free. Recall that $R$ necessarily contains its residue field (see for instance [4, 0, 19.6.3]). Let $\left(f_{i}\right)_{i \in \Lambda}$ be a family of elements of $M$ the images of which form a basis of the $\kappa(R)$-vector space $M / \mathfrak{m}_{R} M$. We will prove that this is a basis of $M$ over $R$. We have to prove that the morphism

$$
\Phi:\left\{\begin{array}{l}
R^{(\Lambda)} \longrightarrow M \\
\left(b_{i}\right)_{i \in \Lambda} \longrightarrow \sum b_{i} f_{i}
\end{array}\right.
$$

is an isomorphism. Owing to Lemma 2.1, we already know that it is surjective. Let us consider a relation $\sum_{i} b_{i} f_{i}=0$. We have to prove that all the $b_{i}$ are zero. Let $\left(e_{1}, \ldots, e_{n}\right)$ be a $\kappa(R)$-basis of $R$ given by the Lemma 3.2. We can write $b_{i}=\sum_{j} \alpha_{i j} e_{j}$ with $\alpha_{i j}$ in $\kappa(R)$. Thus we get the relation

$$
\sum_{i} \sum_{j=1}^{n} \alpha_{i j} e_{j} f_{i}=0 .
$$

Let $a_{1}, \ldots, a_{n-1}$ be the elements of $R$ given together with the basis $\left(e_{1}, \ldots, e_{n}\right)$ in the Lemma 3.2. Multiplying the relation by $a_{1}$, we get:

$$
e_{n} \sum_{i} \alpha_{i 1} f_{i}=0
$$


Since $M$ is weakly torsion-free, we deduce that $\sum_{i} \alpha_{i 1} f_{i}$ belongs to $\mathfrak{m}_{R} M$. But the $f_{i}$ 's form a basis of $M / \mathfrak{m}_{R} M$, so for every $i, \alpha_{i 1}=0$. Proceeding by induction (using $a_{2}, \ldots, a_{n-1}$ ) we prove that all the $\alpha_{i j}$ are equal to zero.

Example 3.8 If we drop the Gorenstein assumption, 3.7 is not true any more. Let $k$ be a field, let $R=k[x, y] /(x, y)^{2}$, and let $M$ be the quotient $R^{2} / I$, where $I$ is the submodule of $R^{2}$ generated by the element $\left(\begin{array}{l}x \\ y\end{array}\right)$. Then $M$ is not flat over $R$ since it is of dimension 5 as a $k$-vector space. But $M$ is weakly torsion-free. Indeed, let $\lambda \in R$ and $m=\left(\begin{array}{l}a \\ b\end{array}\right) \in R^{2}$ such that $\lambda m \in I$. If $\lambda$ is invertible it is obvious that $m \in \mathfrak{m}_{R} R^{2}$. If $\lambda m=0$ it is obvious that either $\lambda=0$ or $m \in \mathfrak{m}_{R} R^{2}$. Now if $\lambda \in \mathfrak{m}_{R}$ and $\lambda m \neq 0$, we have $\lambda a=\alpha x, \lambda b=\alpha y$ with $\alpha \in R^{\times}$ so $a$ and $b$ are invertible and $\lambda \in(x) \cap(y)=(0)$, a contradiction.

Corollary 3.9 Let $\varphi: A \rightarrow B$ be a Gorenstein morphism of Artin local rings. Let $M$ be a $B$-module, flat over $A$. Then the following conditions are equivalent.

(i) The module $M$ is flat over $B$.

(ii) The module $M / \mathfrak{m}_{A} M$ is flat over $B / \mathfrak{m}_{A} B$.

(iii) The module $M / \mathfrak{m}_{A} M$ is weakly torsion-free over $B / \mathfrak{m}_{A} B$.

Proof The equivalence of (i) and (ii) is 2.3. The equivalence of (ii) and (iii) is a consequence of the previous proposition. (Note that the Gorenstein ring $B / \mathfrak{m}_{A} B$ contains the field $\kappa(A)$.)

\section{Flatness in first order}

We begin with a technical lemma that will be used twice: the first time to define the notion of flatness in order one below (4.2 (i)), and the second time in the Proof of Lemma4.11 (that will be useful in Sect. 5 to modify the morphism $\varphi$ when necessary).

Lemma 4.1 Let $A$ be a Noetherian local ring, I an ideal of A, and $M$ an A-module. Let $x=\left(x_{1}, \ldots, x_{n}\right)$ be a sequence generating I minimally. Let us denote by $N_{x}$ the submodule of $M$ generated by the elements $m_{i}$ for all the relations

$$
x_{1} m_{1}+\cdots+x_{n} m_{n}=0 .
$$

Then the module $N_{x}$ does not depend on the choice of $x$.

Proof Let $y=\left(y_{1}, \ldots, y_{n}\right)$ be another sequence generating $I$ minimally. It is enough to prove that $N_{x} \subset N_{y}$. Let

$$
x_{1} m_{1}+\cdots+x_{n} m_{n}=0
$$

be a relation as in the statement of the lemma.

We still write $x$ (resp. $y$ ) for the row matrix $\left(x_{1}, \ldots, x_{n}\right)$ (resp. $\left.\left(y_{1}, \ldots, y_{n}\right)\right)$ and $m$ for the column matrix $\left(m_{1}, \ldots, m_{n}\right)^{t}$. The above relation becomes $x . m=0$. Since $x$ and $y$ are minimal systems of generators of $I$, there is a matrix $C$ of size $n \times n$ with coefficients in $A$ such that $x=y C$. Since the images of $x$ and $y$ in $I / \mathfrak{m}_{A} I$ are both $\kappa(A)$-basis of $I / \mathfrak{m}_{A} I$, this matrix $C$ is invertible modulo $\mathfrak{m}_{A}$. This implies that $C$ is also invertible in $A$ since $A$ is local. The relation $x m=y C m=0$ implies that $C m$ has its entries in $N_{y}$. Hence $m=C^{-1} C m$ also has its entries in $N_{y}$, this proves the assertion. 
Definition 4.2 (i) Let $A$ be a Noetherian local ring and $M$ an $A$-module. Then $M$ is said to be flat in first order (or flat in order one, or simply 1-flat) if for a (resp. for any, see 4.1) minimal system of generators $\left(x_{1}, \ldots, x_{n}\right)$ of $\mathfrak{m}_{A}$, and for elements $m_{1}, \ldots, m_{n}$ in $M$ the relation

$$
x_{1} m_{1}+\cdots+x_{n} m_{n}=0
$$

implies $m_{i} \in \mathfrak{m}_{A} M$ for all $i$. (In other words, if $N_{x} \subset \mathfrak{m}_{A} M$ with the notations of the above lemma.)

(ii) Let $\varphi: A \rightarrow B$ be a morphism of Noetherian local rings and $M$ a $B$-module. Then $M$ is said to be $\varphi$-1-flat if it is 1-flat over $A$ via $\varphi$.

(iii) Let $\varphi: A \rightarrow B$ be a morphism of Noetherian local rings and $M$ be a $B$-module. Then $M$ is said to be $\varphi$-weakly torsion-free if $M / \mathfrak{m}_{A} M$ is a weakly torsion-free $B / \mathfrak{m}_{A} B$ module.

Proposition 4.3 Let $A$ be a Noetherian local ring and $M$ an A-module. Then $M$ is flat in first order if and only if $M / \mathrm{m}_{A}^{2} M$ is flat over $A / \mathfrak{m}_{A}^{2}$. In particular, any flat A-module is 1-flat, and the converse is true if $\mathfrak{m}_{A}^{2}=0$.

Proof Let $\left(e_{i}\right)_{i \in I}$ be an $A / \mathfrak{m}_{A}^{2}$-basis of $M / \mathfrak{m}_{A}^{2} M$. Let $x_{1} m_{1}+\cdots+x_{n} m_{n}=0$ be a relation as in the Definition 4.2 and consider its projection in $M / \mathfrak{m}_{A}^{2} M$. Let us denote by $\lambda_{i j}$ the coefficient of $\overline{m_{j}}$ along $e_{i}$. Then we have the relation in $A / \mathfrak{m}_{A}^{2}$ :

$$
x_{1} \lambda_{i 1}+\cdots+x_{n} \lambda_{i n}=0
$$

Since the $x_{i}$ 's form a basis of $\mathfrak{m}_{A} / \mathfrak{m}_{A}^{2}$ this implies that all the $\lambda_{i j}$ belong to $\mathfrak{m}_{A}$, hence all the $m_{j}$ belong to $\mathfrak{m}_{A} M$.

Conversely, assume that $M$ is 1-flat. Let $\left(e_{i}\right)_{i \in I}$ be a family of elements of $M / \mathfrak{m}_{A}^{2} M$, the images of which form a basis of $M / \mathfrak{m}_{A} M$ over $\kappa(A)$. The $e_{i}$ 's generate $M / \mathfrak{m}_{A}^{2} M$ owing to 2.1 (note that $A / \mathfrak{m}_{A}^{2}$ is Artin). To prove they are linearly independent over $A / \mathfrak{m}_{A}^{2}$, let us consider a relation $\sum \lambda_{i} e_{i}=0$ with $\lambda_{i} \in A$. Viewing the relation in $M / \mathfrak{m}_{A} M$, we immediately see that $\lambda_{i}$ belongs to $\mathfrak{m}_{A}$. Thus we can write $\lambda_{i}=x_{1} \alpha_{1}^{i}+\cdots+x_{n} \alpha_{n}^{i}$ and we have

$$
x_{1}\left(\sum \alpha_{1}^{i} e_{i}\right)+\cdots+x_{n}\left(\sum \alpha_{n}^{i} e_{i}\right)=0 .
$$

Since $M$ is 1-flat, this implies that for all $j, \sum \alpha_{j}^{i} e_{i}=0$ in $M / \mathfrak{m}_{A} M$, thus the $\alpha_{j}^{i}$ belong to $\mathfrak{m}_{A}$ and the $\lambda_{i}$ belong to $\mathfrak{m}_{A}^{2}$.

Remark 4.4 If $\mathfrak{m}_{A}^{2} \neq 0$, a 1-flat module does not need to be flat. For instance in the ring $k[x] /\left(x^{3}\right)$, the ideal $(x)$ is 1-flat but not flat. Actually, since $A / \mathfrak{m}_{A}^{2}$ is 1-flat, we have $\mathfrak{m}_{A}^{2}=0$ if and only if every 1 -flat module is flat.

Definition 4.5 Let $\varphi: A \rightarrow B$ be a morphism of Noetherian local rings. Then $\varphi$ is called nice if every $\varphi$-1-flat $B$-module is $\varphi$-weakly torsion-free.

Remark 4.6 A morphism $\varphi: A \rightarrow B$ of Noetherian local rings is nice if and only if the induced morphism $\varphi \otimes_{A}\left(A / \mathfrak{m}_{A}^{2}\right)$ is nice. (Immediate from the Definitions $4.2,4.5$ and the Proposition 4.3.) Note also that if $\varphi$ is not local then it is obviously nice since $B / \mathfrak{m}_{A} B=0$.

Remark 4.7 If $\varphi$ is a nice Gorenstein morphism of Artin local rings then using 4.3 and 3.9, we see that every $B$-module $M$ that is flat over $A$, is flat over $B$. In particular this proves 1.4. 
Proposition 4.8 Let $\varphi: A \rightarrow B$ be a local morphism of Noetherian local rings and let $M$ be a $B$-module. Assume that $M$ is of finite type over $A$ or that $A$ is Artin. Assume also that $\mu_{B}\left(\mathfrak{m}_{A} B\right)<\operatorname{edim}(A)$, and that $M$ is $\varphi$-1-flat. Then $M=0$.

Proof By 2.1, we only have to prove that $M=\mathfrak{m}_{A} M$. Let $\left(x_{1}, \ldots, x_{n}\right)$ be a minimal system of generators of $\mathfrak{m}_{A}$. By assumption, one of the $\varphi\left(x_{i}\right)$ 's, say $\varphi\left(x_{1}\right)$, is a linear combination of the others:

$$
\varphi\left(x_{1}\right)=\sum_{i=2}^{n} b_{i} \varphi\left(x_{i}\right)
$$

with $b_{i} \in B$. Now if $m$ is an element of $M$, multiplying the above relation by $m$ and using the first order flatness of $M$ over $A$, we see that $m \in \mathfrak{m}_{A} M$.

Corollary 4.9 Let $\varphi: A \rightarrow B$ be a local morphism of Noetherian local rings. Assume that $\mu_{B}\left(\mathfrak{m}_{A} B\right)<\operatorname{edim}(A)$. Then $\varphi$ is nice and de Smit.

Corollary 4.10 Let $\varphi: A \rightarrow B$ be a local complete intersection morphism of Noetherian local rings. Assume that $\operatorname{edim}(B)<\operatorname{edim}(A)$. Then the only $\varphi$-1-flat $B$-module (of finite type over A if A is not Artin) is zero. In particular $\varphi$ is nice and de Smit.

Proof Indeed, using the Lemma 5.4 below, we see that $\mu_{B}\left(\mathfrak{m}_{A} B\right) \leq \operatorname{edim}(B)$. Note that to prove that $\varphi$ is nice, we can reduce to $\mathfrak{m}_{A}^{2}=0$ using 4.6, so $A$ is Artin.

Lemma 4.11 Let $\varphi, \psi: A \rightarrow B$ be two local morphisms of Noetherian local rings. Assume that $\varphi\left(\mathfrak{m}_{A}\right) B=\psi\left(\mathfrak{m}_{A}\right) B$. Let $M$ be a B-module. Then:

(i) $\varphi\left(\mathfrak{m}_{A}\right) M=\psi\left(\mathfrak{m}_{A}\right) M$.

(ii) $M$ is $\varphi$-weakly torsion-free if and only if it is $\psi$-weakly torsion-free.

(iii) $M$ is $\varphi$-1-flat if and only if it is $\psi$-1-flat.

Proof (i) This is obvious.

(ii) This is an immediate consequence of the definition and (i).

(iii) In view of 4.3 , we can assume that $\mathfrak{m}_{A}^{2}=0$, so $A$ is Artin. Now if $\mu_{B}\left(\mathfrak{m}_{A} B\right)<$ $\operatorname{edim}(A)$ the statement is obvious since the only $\varphi$-(resp. $\psi$-) 1 -flat $B$-module is zero, see 4.8. Otherwise, let $\left(x_{1}, \ldots, x_{n}\right)$ be a minimal system of generators of $\mathfrak{m}_{A}$. Then $\left(\varphi\left(x_{1}\right), \ldots, \varphi\left(x_{n}\right)\right)$ and $\left(\psi\left(x_{1}\right), \ldots, \psi\left(x_{n}\right)\right)$ are both minimal systems of generators of $\mathfrak{m}_{A} B$, and the result is a consequence of Lemma 4.1.

\section{Sufficient conditions for the conjecture to be verified}

We will give in this section some particular cases in which we can prove the Conjecture 1.1. The results 5.1, 5.11, 5.12 and 5.13 give the proof of 1.6. We first get rid of the trivial cases of embedding dimension zero or one.

Theorem 5.1 Let $\varphi: A \rightarrow B$ be a morphism of Artin local rings with same embedding dimension $n$ equal to zero or one. Then $\varphi$ is nice.

Proof If $n$ equals 0 , the rings $A$ and $B$ are fields and the statement is obvious. Now assume $n$ is equal to 1 . Let $M$ be a $\varphi$-1-flat $B$-module and let $x$ (resp. $u$ ) be a minimal generator of $\mathfrak{m}_{A}\left(\right.$ resp. $\left.\mathfrak{m}_{B}\right)$. Let $\lambda \in B$ and $m \in M$ be elements such that $\lambda \notin \varphi(x) B$ and $\lambda m \in \varphi(x) M$. 
We have to prove that $m \in u M$. We can assume $\varphi(x) \neq 0$ (see 4.9). Let $p$ (resp. $q$ ) be the greatest integer such that $\varphi(x) \in\left(u^{p}\right)$ (resp. $\lambda \in\left(u^{q}\right)$ ). Then we have $\varphi(x)=a u^{p}$ and $\lambda=b u^{q}$ with $a, b \in B^{\times}$, and $p>q$ since $\lambda$ does not belong to $\varphi(x) B$. There is an $m^{\prime}$ in $M$ such that $\lambda m=\varphi(x) m^{\prime}$. Then:

$$
\begin{aligned}
u^{q}\left(b m-a u^{p-q} m^{\prime}\right) & =0 \\
\varphi(x)\left(b m-a u^{p-q} m^{\prime}\right) & =0 .
\end{aligned}
$$

Since $M$ is $\varphi$-1-flat, this implies that $b m-a u^{p-q} m^{\prime}$ belongs to $\varphi(x) M$, thus $m \in u M$.

Before going further, we recall some facts about Wiebe matrices and consequences of Wiebe's criterion (which recognizes complete intersections of dimension zero among the class of Noetherian local rings).

Definition 5.2 $[11,2.6]$ Let $A$ be a Noetherian local ring with a sequence $x=\left(x_{1}, \ldots, x_{n}\right)$ generating its maximal ideal (not necessarily minimally). An $x$-Wiebe matrix for the $\operatorname{ring} A$ is a square matrix $\psi$ of size $n$ such that $x . \psi=0$ and $\operatorname{det}(\psi) \neq 0$. (Here the sequence $x$ is also viewed as a row matrix.)

Proposition 5.3 (Wiebe, see [11, 2.7]) A Noetherian local ring A is a complete intersection of dimension zero if and only if it has an $x$-Wiebe matrix for some (every) sequence $x$ generating its maximal ideal. When this is the case, the determinant of an x-Wiebe matrix generates the socle of the ring.

Lemma 5.4 Let A be a Noetherian local ring, and I a proper ideal of A. Assume that the ring $A / I$ is a complete intersection.

(i) Then

$$
\mu_{A}(I) \leq \operatorname{edim}(A)-\operatorname{dim}(A / I) .
$$

(ii) If moreover $I \subset \mathfrak{m}_{A}^{2}$, then

$$
\mu_{A}(I) \leq \operatorname{edim}(A / I)-\operatorname{dim}(A / I) .
$$

Remark 5.5 The inequalities are not equalities in general (consider for instance $A=\mathbb{C}[x] /\left(x^{2}\right)$ and $I=0$ ). But if $A$ is regular, then it is well known that (ii) actually is an equality.

Proof We first prove (ii). As the minimal number of generators of any $A$-module is impervious to the change of rings $A \rightarrow \hat{A}$, where $\hat{A}$ is the completion of $A$ in its $\mathfrak{m}_{A}$-adic topology, we are reduced to the case where $A$ is complete. Using Cohen's theory, we know that there exists a regular Noetherian local ring $R$ and a surjective local morphism $\pi: R \rightarrow A$ with $\operatorname{Ker} \pi \subset \mathfrak{m}_{R}^{2}$. Write $J=\pi^{-1}(I)$. Then, since $I \subset \mathfrak{m}_{A}^{2}$ and $\operatorname{Ker} \pi \subset \mathfrak{m}_{R}^{2}$, we have $J \subset \mathfrak{m}_{R}^{2}$. Moreover, by assumption the ring $R / J \simeq A / I$ is a complete intersection. Thus by [10, (21.2)] the ideal $J$ of $R$ is generated by a regular sequence, so that $\mu_{R}(J)=\operatorname{edim}(R / J)-\operatorname{dim}(R / J)$. But $\mu_{A}(I) \leq \mu_{R}(J)$ (because $\pi$ is surjective). This gives the result.

Now let us prove (i), using (ii). The inclusion of the ideal $I$ in $\mathfrak{m}_{A}$ induces a map of $\kappa(A)$ vector spaces $\psi: \frac{I}{\mathfrak{m}_{A} I} \rightarrow \frac{\mathfrak{m}_{A}}{\mathfrak{m}_{A}^{2}}$. Let $x_{1}, \ldots, x_{i}$ be a sequence of elements of $I$, the images of which form a basis of the image of $\psi$. We complete it to form a minimal system of generators $\left(x_{1}, \ldots, x_{n}\right)$ of $\mathfrak{m}_{A}$. Let $J \subset I$ be the ideal generated by the elements $x_{1}, \ldots, x_{i}$. Let us denote by $A^{\prime}$ the quotient ring $A / J$ and $I^{\prime}$ the ideal $I / J$ in $A^{\prime}$. Then we see that $I^{\prime} \subset \mathfrak{m}_{A^{\prime}}^{2}$, and the ring $A^{\prime} / I^{\prime} \simeq A / I$ is a complete intersection. Thus using (ii), we get

$$
\begin{aligned}
\mu_{A^{\prime}}\left(I^{\prime}\right) & \leq \operatorname{edim}\left(A^{\prime} / I^{\prime}\right)-\operatorname{dim}\left(A^{\prime} / I^{\prime}\right) \\
& \leq \operatorname{edim}\left(A^{\prime}\right)-\operatorname{dim}(A / I)
\end{aligned}
$$


Moreover, we obviously have

$$
\mu_{A}(I) \leq \mu_{A}(J)+\mu_{A}\left(I^{\prime}\right) .
$$

Now we have $\mu_{A}(J) \leq i$ by definition of $J$, and $\mu_{A}\left(I^{\prime}\right)=\mu_{A^{\prime}}\left(I^{\prime}\right)$. We conclude

$$
\begin{aligned}
\mu_{A}(I) & \leq i+\operatorname{edim}\left(A^{\prime}\right)-\operatorname{dim}(A / I) \\
& \leq \operatorname{edim}(A)-\operatorname{dim}(A / I)
\end{aligned}
$$

The existence of an element $u \in B$ verifying the equivalent conditions (i)-(vi) given in the following lemma is precisely the condition under which we (the authors) can go from embedding dimension $n$ to embedding dimension $n-1$ when trying to prove de Smit's Conjecture 1.6 (see 5.7 and the proof of 5.13). Unfortunately such an element $u$ does not always exist (see 6.1.3). Note that, as mentioned in the introduction, there is a Maple program on the webpage of the first author for testing (in some cases) if such an element exists.

Lemma 5.6 Let $\varphi: A \rightarrow B$ be a complete intersection morphism of Artin local rings. Assume that

$$
\operatorname{edim}(A)=\operatorname{edim}(B)=\operatorname{dim}_{\kappa(B)} \frac{\mathfrak{m}_{A} B}{\mathfrak{m}_{B} \mathfrak{m}_{A} B} .
$$

Let $n$ denote this dimension and $B_{0}$ denote the quotient ring $B / \mathfrak{m}_{A} B$. Let $u$ be a minimal generator of $\mathfrak{m}_{B}$ such that its image $u_{0}$ in $B_{0}$ is a minimal generator of $\mathfrak{m}_{B_{0}}$ (i.e. $u$ is not in $\left.\mathfrak{m}_{A} B+\mathfrak{m}_{B}^{2}\right)$. Let $I=\operatorname{Ann}\left(u_{0}\right)$ denote the annihilator of $u_{0}$. The following are equivalent:

(i) There is an element $y$ in $\mathfrak{m}_{A} B \backslash \mathfrak{m}_{B} \mathfrak{m}_{A} B$ such that $u$ divides $y$ in $B$.

(ii) The ring $B_{0} /\left(u_{0}\right)$ is a complete intersection.

(iii) The ring $B_{0} / I$ is a complete intersection.

(iv) The ideal I of $B_{0}$ is principal.

(v) For any (for some) sequence $v=\left(v_{1}, \ldots, v_{r}\right)$ generating $\mathfrak{m}_{B_{0}}$ (not necessarily minimally), there is a $v$-Wiebe matrix $\psi$ of $B_{0}$ such that its entries in the first column are all multiples of $u$.

(vi) For any (for some) sequence $v=\left(u_{0}, v_{2}, \ldots, v_{n}\right)$ generating $\mathfrak{m}_{B_{0}}$ minimally, there is a $v$-Wiebe matrix for $B_{0}$ of the form

$$
\psi=\left(\begin{array}{c|c}
z_{1} & \ldots \\
\hline 0 & \psi^{*}
\end{array}\right) .
$$

Moreover if these conditions hold, and if $\psi$ is a matrix as in (vi), then $\psi^{*}$ is a $\left(v_{2}, \ldots, v_{n}\right)$ Wiebe matrix for the ring $B_{0} /\left(u_{0}\right)$.

Proof The equivalence of conditions (ii) to (vi) and the last statement are proved in [11, 5.5 and 5.7]. So we only have to prove the equivalence of (i) and (ii).

Assume (i). Using Cohen's theory, there is a Noetherian regular local ring $R$ and a surjective local morphism $\pi: R \rightarrow B$, with $\operatorname{Ker} \pi \subset \mathfrak{m}_{R}^{2}$. In $R$, there are liftings $y^{\prime}$ and $u^{\prime}$ of $y$ and $u$ such that $y^{\prime}$ is a multiple of $u^{\prime}$. Let $J=\pi^{-1}\left(\mathfrak{m}_{A} B\right)$. Since $\pi\left(\mathfrak{m}_{R}\right) \subset \mathfrak{m}_{B}$, we easily see that $y^{\prime}$ and $u^{\prime}$ are minimal generators of $J$ and $\mathfrak{m}_{R}$ respectively. Let $\left(y^{\prime}, y_{2}, \ldots, y_{s}\right)$ be a minimal system of generators of $J$. This is a regular sequence since the ring $R / J$, isomorphic to $B_{0}$, is a complete intersection. This means that the sequence $\left(y_{2}, \ldots, y_{s}\right)$ is regular and that $y^{\prime}$ is not a zero divisor in $R /\left(y_{2}, \ldots, y_{s}\right)$. Neither is $u^{\prime}$ since $y^{\prime}$ is a multiple of $u^{\prime}$. Thus 
$\left(u^{\prime}, y_{2}, \ldots, y_{s}\right)$ is a regular sequence and the ring $R /\left(u^{\prime}, y_{2}, \ldots, y_{s}\right)=R /\left(J+\left(u^{\prime}\right)\right)$ is a complete intersection. This last ring is isomorphic to $B_{0} /\left(u_{0}\right)$, proving (ii).

Conversely, assume (ii). Let $\pi$ be the projection map $\pi: B \rightarrow \bar{B}=B /(u)$. The quotient ring $\bar{B} / \mathfrak{m}_{A} \bar{B}$ is isomorphic to $B_{0} /\left(u_{0}\right)$, and so is a complete intersection. Applying the previous Lemma 5.4 to $\bar{B}$ and its ideal $\mathfrak{m}_{A} \bar{B}$, we get

$$
\mu_{\bar{B}}\left(\mathfrak{m}_{A} \bar{B}\right) \leq \operatorname{edim}(\bar{B})=n-1
$$

Let $\left(x_{1}, \ldots, x_{n}\right)$ be a sequence in $A$ generating $\mathfrak{m}_{A}$ minimally. With the above, the sequence $\pi\left(\varphi\left(x_{1}\right)\right), \ldots, \pi\left(\varphi\left(x_{n}\right)\right)$ in $\bar{B}$, which generates $\mathfrak{m}_{A} \bar{B}$, does not generate $\mathfrak{m}_{A} \bar{B}$ minimally. Thus one of the $\pi\left(\varphi\left(x_{i}\right)\right)$, say $\pi\left(\varphi\left(x_{1}\right)\right)$, is a linear combination of the others and we have in $\bar{B}$ a relation $\pi\left(\varphi\left(x_{1}\right)+\alpha_{2} \varphi\left(x_{2}\right)+\cdots+\alpha_{n} \varphi\left(x_{n}\right)\right)=0$ for some $\alpha_{i} \in B$. Now the element $y=\varphi\left(x_{1}\right)+\alpha_{2} \varphi\left(x_{2}\right)+\cdots+\alpha_{n} \varphi\left(x_{n}\right)$ is in $\mathfrak{m}_{A} B \backslash \mathfrak{m}_{B} \mathfrak{m}_{A} B$ and in Ker $\pi=(u)$.

Lemma 5.7 Let $\varphi: A \rightarrow B$ be a morphism of Artin local rings. Assume that there is a minimal generator $u$ of $\mathfrak{m}_{B}$ and a minimal generator $x$ of $\mathfrak{m}_{A}$ such that $\varphi(x)$ is a multiple of $u$ and does not belong to $\mathfrak{m}_{B} \mathfrak{m}_{A} B$. Let us denote by

$$
\bar{\varphi}: \bar{A}=A /(x) \rightarrow B /(u)=\bar{B}
$$

the induced morphism of Artin local rings.

(i) If $\varphi$ is a complete intersection morphism then so is $\bar{\varphi}$.

(ii) If $\mathfrak{m}_{A}^{2}=0$, then $\mathfrak{m}_{\frac{2}{A}}^{2}=0$.

(iii) The embedding dimensions of $\bar{A}$ and $\bar{B}$ are respectively edim $(A)-1$ and edim $(B)-1$. In particular, if $A$ and $B$ have same embedding dimension, then so have $\bar{A}$ and $\bar{B}$.

(iv) If $\mathfrak{m}_{A} B / \mathfrak{m}_{A}^{2} B$ is free of rank edim (A) over $B / \mathfrak{m}_{A} B$, then $\mathfrak{m}_{\bar{A}} \bar{B} / \mathfrak{m}_{\bar{A}} \frac{2}{B}$ is free of rank edim $(\bar{A})$ over $\bar{B} / \mathfrak{m}_{\bar{A}} \bar{B}$. (The interest of this property is that it implies that $\operatorname{dim}_{\kappa(B)} \mathfrak{m}_{A} B / \mathfrak{m}_{B} \mathfrak{m}_{A} B=\operatorname{edim}(A)$.)

(v) If $M$ is a $\varphi$-1-flat $B$-module, then $\bar{M}:=M / u M$ is a $\bar{\varphi}$-1-flat $\bar{B}$-module.

(vi) If $B / \mathfrak{m}_{A} B$ is Gorenstein and if $\bar{\varphi}$ is nice, then $\varphi$ is nice.

Proof (ii) and (iii) are obvious. (i) is a consequence of the implication $(i) \Rightarrow$ (ii) of 5.6, taking $\varphi(x)$ for the element $y$.

(iv) Let $n=\operatorname{edim}(A)$ and let $\left(x_{1}, \ldots, x_{n}\right)$ be a minimal system of generators of $\mathfrak{m}_{A}$, with $x_{1}=x$. We will prove that the images of the elements $\left(\varphi\left(x_{2}\right), \ldots, \varphi\left(x_{n}\right)\right)$ in $\mathfrak{m}_{A} \bar{B} / \mathfrak{m}_{\bar{A}} \frac{2}{B}$ form a basis of this module over $\bar{B} / \mathfrak{m}_{\bar{A}} \bar{B}$. We only have to prove that they are linearly independant. So let $\alpha_{2}, \ldots, \alpha_{n}$ be elements of $B$ such that the image of $\alpha_{2} \varphi\left(x_{2}\right)+\cdots+\alpha_{n} \varphi\left(x_{n}\right)$ in the module $\mathfrak{m}_{\bar{A}} \bar{B} / \mathfrak{m}_{\bar{A}} \frac{2}{B}$ is zero, and let us prove that for all $i, \overline{\alpha_{i}}$ belongs to $\mathfrak{m}_{A} \bar{B}$, i.e. that $\alpha_{i}$ belongs to $\mathfrak{m}_{A} B+(u)$. By assumption, we have

$$
\sum_{i=2}^{n} \alpha_{i} \varphi\left(x_{i}\right) \in(u)+\mathfrak{m}_{A}^{2} B .
$$

We multiply this by an element $d \in B$ such that $d u=\varphi(x)$ and obtain

$$
\sum_{i=2}^{n} d \alpha_{i} \varphi\left(x_{i}\right) \in(\varphi(x))+\mathfrak{m}_{A}^{2} B .
$$


But, with the assumption in (iv), the images of the $\varphi\left(x_{i}\right)$ 's in $\mathfrak{m}_{A} B / \mathfrak{m}_{A}^{2} B$ form a basis of this free module over $B / \mathfrak{m}_{A} B$, so that $d \alpha_{i} \in \mathfrak{m}_{A} B$ for all $i$. Hence we can write:

$$
d \alpha_{i}=\sum_{j=1}^{n} \beta_{i j} \varphi\left(x_{j}\right)
$$

with $\beta_{i j} \in B$. Multiplying by $u$, we get:

$$
\begin{array}{r}
\varphi(x) \alpha_{i}=\sum_{j=1}^{n} \beta_{i j} u \varphi\left(x_{j}\right) \\
\varphi(x)\left(\alpha_{i}-u \beta_{i 1}\right)=\sum_{j=2}^{n} \beta_{i j} u \varphi\left(x_{j}\right)
\end{array}
$$

We remember that the images of the $\varphi\left(x_{i}\right)^{\prime}$ 's in $\mathfrak{m}_{A} B / \mathfrak{m}_{A}^{2} B$ form a basis of this free $B / \mathfrak{m}_{A} B$ module, and we conclude that $\alpha_{i}-u \beta_{i 1} \in \mathfrak{m}_{A} B$.

(v) Let $M$ be a $\varphi$-1-flat $B$-module and let us view $M$ as an $A$-module via $\varphi$. Let $\left(x, x_{2}\right.$, $\left.\ldots, x_{n}\right)$ be a minimal system of generators of $\mathfrak{m}_{A}$. Then $\left(\overline{x_{2}}, \ldots, \overline{x_{n}}\right)$ is a minimal system of generators of $\mathfrak{m}_{\bar{A}}$. Let

$$
\overline{x_{2} m_{2}}+\cdots+\overline{x_{n} m_{n}}=0
$$

be a relation in $\bar{M}$ viewed as an $\bar{A}$-module via $\bar{\varphi}$. We then have

$$
x_{2} m_{2}+\cdots+x_{n} m_{n}=u m_{1}
$$

in $M$. Since $M$ is $\varphi$-1-flat, since $\varphi(x)=d u$ for some $d \in B$, this implies that $m_{1}$ belongs to $\mathfrak{m}_{A} M$, i.e. $m_{1}=x_{1} m_{1}^{\prime}+\cdots+x_{n} m_{n}^{\prime}$. We get:

$$
x_{2}\left(m_{2}-u m_{2}^{\prime}\right)+\cdots+x_{n}\left(m_{n}-u m_{n}^{\prime}\right)=u x_{1} m_{1}^{\prime}
$$

thus $m_{i}-u m_{i}^{\prime} \in \mathfrak{m}_{A} M$ for $i \geq 2$ using 1 -flatness again. This proves that $\overline{m_{i}}$ belongs to $\mathfrak{m}_{\bar{A}} \bar{M}$.

(vi) Let $M$ be a $\varphi$-1-flat $B$-module. Let $v \in B$ and $m \in M$ such that $v m \in \mathfrak{m}_{A} M$ and $v \notin \mathfrak{m}_{A} B$. We want to prove that $m \in \mathfrak{m}_{B} M$. Plainly we may assume that $v$ modulo $\mathfrak{m}_{A} B$ generates the socle of $B / \mathfrak{m}_{A} B$. We write $\varphi(x)=u d$, with $d \in B$. The element $d$ is not in $\mathfrak{m}_{A} B$ (otherwise $\varphi(x)$ would belong to $\mathfrak{m}_{B} \mathfrak{m}_{A} B$ ). Thus $(v) \subset(d)$ in $B / \mathfrak{m}_{A} B$ and there is a $b \in B$ and $a \in \mathfrak{m}_{A} B$ such that $v=d b+\lambda$. Now $d b m \in \mathfrak{m}_{A} M$, thus

$$
\begin{gathered}
d b m=u d m_{1}+\varphi\left(x_{2}\right) m_{2}+\cdots+\varphi\left(x_{n}\right) m_{n} . \\
u d\left(b m-u m_{1}\right)=\varphi\left(x_{2}\right) u m_{2}+\cdots+\varphi\left(x_{n}\right) u m_{n}
\end{gathered}
$$

Since $M$ is $\varphi$-1-flat, this implies that $b m-u m_{1}$ belongs to $\mathfrak{m}_{A} M$. Hence the element $\bar{b} \bar{m}$ of $\bar{M}$ belongs to $\mathfrak{m}_{\bar{A}} \bar{M}$. But $\bar{b}$ does not belong to $\mathfrak{m}_{\bar{A}} \bar{B}$ (otherwise $d b$ would belong to $\mathfrak{m}_{A} B$ since $d u$ does). Moreover we have seen that $\bar{M}$ is $\bar{\varphi}-1$-flat so that $\bar{M} / \mathfrak{m}_{\bar{A}} \bar{M}$ is a weakly torsion-free $\bar{B} / \mathfrak{m}_{\bar{A}} \bar{B}$-module since $\bar{\varphi}$ is nice. Thus $\bar{m}$ belongs to $\mathfrak{m}_{\bar{B}} \bar{M}$. This easily implies that $m \in \mathfrak{m}_{B} M$.

Lemma 5.8 Let $\varphi: A \rightarrow B$ be a morphism of Artin local rings. Assume that $\mathfrak{m}_{A}^{2}=0$. Let $\left(x_{1}, \ldots, x_{n}\right)$ be a minimal system of generators of $\mathfrak{m}_{A}$, and $\left(y_{1}, \ldots, y_{n}\right)$ a system of generators of $\varphi\left(\mathfrak{m}_{A}\right) B$ (not necessarily minimal). In case A does not contain a field, we assume moreover that $x_{1}=p \cdot 1_{A}$ and $y_{1}=p \cdot 1_{B}$, where $p$ is the characteristic of $\kappa(A)$. Then: 
(i) There is a morphism $\psi: A \rightarrow B$ such that $\psi\left(x_{i}\right)=y_{i}$.

(ii) For such a morphism, the ideals $\varphi\left(\mathfrak{m}_{A}\right) B$ and $\psi\left(\mathfrak{m}_{A}\right) B$ are equal.

Remark 5.9 If $A$ does not contain a field, then the characteristic $p$ of $\kappa(A)$ is different from zero and we have $p .1_{A} \neq 0[4$, chap. $0,19.6 .3]$, so that $p .1_{A}$ is a minimal generator of $\mathfrak{m}_{A}$ since $\mathfrak{m}_{A}^{2}=0$. Thus the additional hypothesis $x_{1}=p$ and $y_{1}=p$ is not a restriction on $\varphi$ but only on the choice of the minimal system of generators of $\mathfrak{m}_{A}$.

Proof (i) If $A$ contains a field, then it contains $\kappa(A)$ and the map $T_{i} \mapsto x_{i}$ defines an isomorphism from

$$
\frac{\kappa(A)\left[\left|T_{1}, \ldots, T_{n}\right|\right]}{\left(T_{1}, \ldots, T_{n}\right)^{2}}
$$

to $A$ (we use $\mathfrak{m}_{A}^{2}=0$ ). Via this isomorphism, and since $\left(\mathfrak{m}_{A} B\right)^{2}=0$, we can obviously define $\psi$ by $T_{i} \mapsto y_{i}$.

If $A$ does not contain a field, let $W_{A}$ be a Cohen ring, the residue field of which is $\kappa(A)$. $W_{A}$ is a discrete valuation ring, and its maximal ideal is generated by $p$. Since $\mathfrak{m}_{A}^{2}=0$, we have an isomorphism

$$
A \simeq \frac{W_{A}\left[\left|T_{2}, \ldots, T_{n}\right|\right]}{\left(p, T_{2}, \ldots, T_{n}\right)^{2}}
$$

identifying $T_{i}$ with $x_{i}(2 \leq i \leq n)$ and we can again define the map $\psi$ sending $T_{i}$ to $y_{i}$.

(ii) Obvious by construction.

The following lemma prepares the proof of the Conjecture 1.1 in embedding dimension 2.

Lemma 5.10 Let $\varphi: A \rightarrow B$ be a morphism of Artin local rings of embedding dimension 2. Assume that

$$
\operatorname{dim}_{\kappa(B)} \frac{\mathfrak{m}_{A} B}{\mathfrak{m}_{B} \mathfrak{m}_{A} B}=2 .
$$

Then for every minimal generator $u$ of $\mathfrak{m}_{B}$, there is an element $y$ in $\mathfrak{m}_{A} B \backslash \mathfrak{m}_{B} \mathfrak{m}_{A} B$ such that $u$ divides $y$ in $B$.

Proof Let $(u, v)$ be a minimal system of generators of $\mathfrak{m}_{B}$ and $(x, y)$ a minimal system of generators of $\mathfrak{m}_{A} B$ (i.e. a basis of $\frac{\mathfrak{m}_{A} B}{\mathfrak{m}_{B} \mathfrak{m}_{A} B}$ ). We can write

$$
\begin{aligned}
& x=\alpha v^{n}+u b_{1} \\
& y=\beta v^{m}+u b_{2}
\end{aligned}
$$

with $b_{1}, b_{2} \in B$ and $\alpha, \beta \in B^{\times}$. We can assume $m \geq n$. Then $y-\beta \alpha^{-1} v^{m-n} x$ is a multiple of $u$, and is an element of $\mathfrak{m}_{A} B \backslash \mathfrak{m}_{B} \mathfrak{m}_{A} B$.

Theorem 5.11 Let $\varphi: A \rightarrow B$ be a Gorenstein morphism of Artin local rings of embedding dimension two. Then $\varphi$ is nice.

Proof Using 4.9 and 4.6, we can assume that

$$
\operatorname{dim}_{\kappa(B)} \frac{\mathfrak{m}_{A} B}{\mathfrak{m}_{B} \mathfrak{m}_{A} B}=2
$$

and that $\mathfrak{m}_{A}^{2}=0$. Let $u$ be a minimal generator of $\mathfrak{m}_{B}$ and $y$ given by the previous lemma. Using Lemma 5.8 there is a morphism $\psi: A \rightarrow B$ such that $\psi(x)=y$ for a minimal 
generator $x$ of $\mathfrak{m}_{A}$ and $\psi\left(\mathfrak{m}_{A}\right) B=\varphi\left(\mathfrak{m}_{A}\right) B$. Using Lemma 4.11, we may replace $\varphi$ with $\psi$ so that we may assume $\varphi(x)=y$. Now owing to Theorem 5.1, the induced morphism $\bar{\varphi}: A /(x) \rightarrow B /(u)$ is nice. Hence $\varphi$ is nice because of Lemma 5.7 (vi).

Remark 5.12 The same argument proves theorem 1.6 (ii).

Theorem 5.13 Let $\varphi: A \rightarrow B$ be a morphism of Artin local rings with same embedding dimension. Write $B_{0}=B / \mathfrak{m}_{A} B$ and assume that for some minimal system of generators $u=\left(u_{1}, \ldots, u_{n}\right)$ of $\mathfrak{m}_{B_{0}}$, there is an upper triangular $u$-Wiebe matrix for $B_{0}$. Then $\varphi$ is nice.

Proof Note that, since there is a $u$-Wiebe matrix for $B_{0}, \varphi$ is a complete intersection morphism (5.3). Proceeding by induction on $n=\operatorname{edim}(A)$ (5.7), and using 5.1 for the initial step, we may assume (and we do) that the theorem is true in embedding dimension $n-1$. We can also assume $(4.9,4.6)$ that

$$
\operatorname{dim}_{\kappa(B)} \frac{\mathfrak{m}_{A} B}{\mathfrak{m}_{B} \mathfrak{m}_{A} B}=\operatorname{edim}(A)
$$

and that $\mathfrak{m}_{A}^{2}=0$. Let $u^{\prime}$ be a lifting of $u_{1}$ in $B$. Owing to Lemma 5.6, there is an element $y$ in $\mathfrak{m}_{A} B \backslash \mathfrak{m}_{B} \mathfrak{m}_{A} B$ such that $u^{\prime}$ divides $y$ in $B$. Using Lemma5.8 there is a morphism $\psi: A \rightarrow B$ sending a minimal generator $x$ of $\mathfrak{m}_{A}$ on $y$ and such that $\psi\left(\mathfrak{m}_{A}\right) B=\varphi\left(\mathfrak{m}_{A}\right) B$. We may replace $\varphi$ by $\psi$ (4.11) so that we may assume $\varphi(x)=y$. Now the induced morphism $\bar{\varphi}: A /(x) \rightarrow B /(u)$ is nice (use Lemma 5.6 and the induction hypothesis). Hence $\varphi$ is nice because of Lemma 5.7 .

Remark 5.14 The question of existence of such upper triangular Wiebe matrices has been considered in [11].

\section{Examples and further developments}

\subsection{Examples}

Example 6.1.1 Let us consider the morphism

$$
\varphi: A=\frac{\left(\mathbb{Z} / p^{2} \mathbb{Z}\right)\left[x_{1}, x_{2}, x_{3}\right]}{\left(x_{1}^{2}, x_{2}^{2}, x_{3}^{2}\right)} \longrightarrow B=\frac{\left(\mathbb{Z} / p^{2} \mathbb{Z}\right)[t, u, v, w]}{\left(t^{2}-p, \alpha_{1}^{2}, \alpha_{2}^{2}, \alpha_{3}^{2}\right)}
$$

where $\varphi$ is defined by $\varphi\left(x_{i}\right)=\alpha_{i}$ and

$$
\begin{aligned}
& \alpha_{1}=u^{2}+t w^{2} \\
& \alpha_{2}=v^{2}+t^{3}+u^{3} \\
& \alpha_{3}=w^{3}+v w
\end{aligned}
$$

The rings $A$ and $B$ are Artin local with embedding dimension 4. We let the reader check that the (image in $B_{0}$ of the) matrix

$$
\left(\begin{array}{cccc}
t & w^{2} & t^{2} & 0 \\
0 & u & u^{2} & 0 \\
0 & 0 & v & w \\
0 & 0 & 0 & w^{2}
\end{array}\right)
$$


is a $(t, u, v, w)$-Wiebe matrix for the ring $B_{0}=B / \mathfrak{m}_{A} B \simeq \frac{\mathbb{F}_{p}[t, u, v, w]}{\left(t^{2}, \alpha_{1}, \alpha_{2}, \alpha_{3}\right)}$. (Compute a Gröbner basis and check that $t u v w^{2}$ is not zero in $B_{0}$.) Hence we deduce from Theorem 5.13 that any $A$-flat $B$-module is $B$-flat.

Example 6.1.2 Let us consider

$$
\begin{aligned}
& p_{1}=u^{2}+v w \\
& p_{2}=v^{2}+u w \\
& p_{3}=w^{2}+u v
\end{aligned}
$$

and the morphism $\varphi: A=\frac{\mathbb{C}\left[\left[x_{1}, x_{2}, x_{3}\right]\right]}{\left(x_{1}, x_{2}, x_{3}\right)^{2}} \rightarrow B=\frac{\mathbb{C}[[u, v, w]]}{\left(p_{1}, p_{2}, p_{3}\right)^{2}}$ defined by $\varphi\left(x_{i}\right)=p_{i}$. We let the reader check that $\varphi$ is flat $\left(\right.$ e.g. $1, w, w^{2}, w^{3}, v, v w, v^{2}, u$ is an $A$-basis of $\left.B\right)$. Hence $B / \mathfrak{m}_{A} B$ is a complete intersection owing to 2.5. Moreover, the element $u-v$ is a minimal generator of $\mathfrak{m}_{B}$, and divides the element $p_{1}-p_{2}$ of $\mathfrak{m}_{A} B \backslash \mathfrak{m}_{B} \mathfrak{m}_{A} B$. Thus using 1.6 (ii) we see that every $A$-flat $B$-module is $B$-flat.

Example 6.1.3 Now let us try with

$$
\begin{aligned}
& p_{1}=u^{3}+v w \\
& p_{2}=v^{2}+u w^{2} \\
& p_{3}=w^{3}+u^{2} v
\end{aligned}
$$

We can check (with the help of a computer) that the condition given in 1.6 (ii) is not true for $\varphi$. Hence de Smit's question remains open in this case.

\subsection{Further developments}

For $A \rightarrow B$ a flat morphism of local rings, Lech [8] and Hironaka [7] asked whenever we have inequality of $i$-th sum transforms of the Hilbert series of $A$ and of $B$ (see [6]). This question is a generalization of the inequality of the embedding dimensions (edim $(A) \leq \operatorname{edim}(B))$. We could discuss a weaker version of de Smit's conjecture using these transforms of Hilbert series. In view of 1.6 it is also tempting to ask whether a morphism of Artin local rings with the same embedding dimension is always nice.

De Smit's question has origin in Wiles' proof of Fermat's theorem ([13]). Wiles' proof needs an isomorphism between an Hecke algebra and a deformation ring. De Smit mentionned his conjecture in order to make easier one argument in this direction ([9]). But such a criterion will not only have applications in deformation theory but also in many other fields. For example let $R$ be a regular ring of characteristic $p$ and $F: R \rightarrow R$ the Frobenius. Consider $F^{*} R$ the $(R-R)$ bimodule with additive group $R$ and left and right scalar multiplication given by $\operatorname{ar} b=\operatorname{ar} F(b)$ for $a, b \in R$ and $r \in F^{*} R$. Then it is well known that $R \rightarrow F^{*} R$ is a flat couple ([3] Sect. 8.2). Then such a flatness criterium could also have some applications in the theory of $\varphi$-module of Fontaine in order to study $R$ and $F^{*} R$-modules.

Acknowledgments We would like to thank Bart de Smit for raising his interesting conjecture to our attention. We also thank the referee for reading very carefully our paper and for numerous valuable suggestions.

Open Access This article is distributed under the terms of the Creative Commons Attribution Noncommercial License which permits any noncommercial use, distribution, and reproduction in any medium, provided the original author(s) and source are credited. 


\section{References}

1. Atiyah, M., MacDonald, I.: Introduction to commutative algebra. Addison-Wesley, Englewood Cliffs (1969)

2. Bourbaki, N.: Algèbre commutative. Masson, Paris

3. Bruns, W., Herzog, J.: Cohen-Macaulay rings. Cambridge studies in advanced mathematics, vol. 39 (1993)

4. Grothendieck, A.: Éléments de géométrie algébrique. IV. Étude locale des schémas et des morphismes de schémas. Inst. Hautes Études Sci. Publ. Math. 20, 24, 28, 32 (1964-1967)

5. Hartshorne, R.: Algeabraic geometry. Graduate Texts in Mathematics, vol. 52. Springer (1977)

6. Herzog, B.: Lech-Hironaka inequalities for flat couples of local rings. Manuscr. Math 68, 351-371 (1990)

7. Hironaka, H.: Certain numerical characters of singularities. J. Math. Kyoto Uni. 151-187 (1970)

8. Lech, C.: Inequalities related to certain couples of local rings. Acta Math. 112, 69-89 (1964)

9. de Smit, B., Rubin, K., Schoof, R.: Criteria for complete intersections. In: Cornell, G., Silverman, J., Stevens, G. (eds.) Modular Forms and Fermat's Last Theorem, pp. 343-355. Springer, Berlin (1997)

10. Matsumura, H.: Commutative Ring Theory. Cambridge University Press, London (1986)

11. Simon, A.-M., Strooker, J.: Complete intersections of dimension zero: variations on a theme of Wiebe. Preprint Arxiv 0703880 (2006)

12. Vasconcelos, W.: Ideals generated by $R$-sequences. J. Algebra 6, 309-316 (1967)

13. Wiles, A.: Modular elliptic curves and Fermat's last theorem. Ann. Math. 141, 443-551 (1995) 